Cómo citar este trabajo: Segura Calero, S. (2018). Planificación comprehensiva en Estados Unidos: evolución en el caso de Arizona y del condado de Pima. Boletín de la Asociación de Geógrafos Españoles, 79, 2520, 1-26. http://dx.doi.org/10.21138/bage.2520a

\title{
Planificación comprehensiva en Estados Unidos: evolución en el caso de Arizona y del condado de Pima
}

Urban comprehensive planning in the United States: the case of Arizona and Pima County

\author{
Sergio Segura Calero \\ ssegura@us.es \\ Departamento de Geografía Humana \\ Universidad de Sevilla (España)
}

\section{Resumen}

Este artículo presenta la planificación comprehensiva territorial en Estados Unidos y profundiza sobre el impacto de las teorías del crecimiento inteligente en el caso de Arizona. El análisis de los planes comprehensivos del condado de Pima, con el apoyo de entrevistas, permite comparar experiencias de planificación territorial. Las conclusiones destacan avances de esta experiencia paralela a la ordenación del territorio española y europea donde, tras los conflictos y problemas detectados, se aboga por una planificación menos tecnocrática y más participada.

Palabras clave: ordenación del territorio; planificación comprehensiva territorial; Estados Unidos; Arizona.

\begin{abstract}
In an instructive way, the theoretical framework of urban comprehensive planning in the United States is presented. Emphasis on the impact of the Smart Growth policies in many American states is placed, such as Arizona in the 1990s. The analysis of the urban comprehensive plans, supported by interviews with experts, allows to get deep into the territorial governance and social innovation
\end{abstract}


planning methodologies. The conclusions of this article show new efforts in citizen participation and good practices to take into account for spatial planning in Spain and Europe.

Key words: spatial planning; comprehensive planning; territorial planning; United States of America; Arizona.

\section{Introducción}

Múltiples estudios relacionados con el sistema de planificación territorial en Estados Unidos muestran diversas tradiciones y situaciones en cada estado. La Asociación Americana de Planificación o el Instituto Lincoln de Políticas de Suelo cuentan con numerosos trabajos y publicaciones que lo demuestran. Dentro de esta diversidad, Pendall y Puentes (2008) identificaron tres modelos de gobernanza territorial distintos en áreas metropolitanas americanas. Uno de ellos toma al Estado de Texas como paradigma de planificación de usos del suelo desregularizada basada en el mercado libre del suelo. Otro enfatiza el localismo de la toma de decisiones en la planificación metropolitana de los estados del interior que han tenido un menor crecimiento urbano. Por último, generalmente en los estados costeros, se identifica un sistema de planificación más avanzado con base en las teorías del crecimiento inteligente. El artículo se centra en este último modelo de planificación, y en la tradición de la planificación comprehensiva y las teorías del crecimiento inteligente de los años noventa en el estado de Arizona.

Este trabajo tiene el objetivo de presentar e identificar elementos y buenas prácticas en la planificación comprehensiva americana que resulten útiles en la ordenación del territorio europea y española. Primeramente el artículo cuenta con un apartado introductorio que define y presenta de forma resumida las bases de la planificación comprehensiva en Estados Unidos. Posteriormente el trabajo se centra en el estado de Arizona, influenciado de forma importante por las teorías del crecimiento inteligente de los años ochenta y noventa. Además, se realiza un análisis práctico de los planes comprehensivos y de su implementación en el condado de Pima y en la aglomeración urbana de Tucson. Un último apartado de conclusiones sintetiza las aportaciones más relevantes del análisis.

Desde el punto de vista metodológico, el marco teórico de este trabajo ha supuesto la revisión de una amplia literatura científica y de la normativa que acompaña a la planificación comprehensiva desde inicios del siglo XX en Estados Unidos hasta la actualidad. También han sido estudiados en profundidad los artículos dedicados a la planificación comprehensiva en los Estatutos Revisados del estado de Arizona, así como los documentos de los planes comprehensivos del condado de Pima y de la aglomeración urbana de la ciudad de Tucson. Asimismo, para realizar el análisis de la situación en la práctica, han sido llevadas a cabo un total de cuatro entrevistas de una hora a técnicos de los departamentos de planificación del condado de Pima y de la ciudad de Tucson. Se 
ha seguido la técnica de muestreo no probabilística snowball para identificar a los actores más relevantes para este estudio. Se trata de una técnica ampliamente utilizada en estudios de ciencias sociales. Las entrevistas han sido consideradas como anónimas para favorecer la libertad de las respuestas a cuestiones abiertas (Tabla 1) que permiten conocer el grado de implementación en la práctica de los planes comprehensivos y la problemática subyacente.

\section{Tabla 1. Cuestionario de entrevistas}

\begin{tabular}{|c|}
\hline Entrevistas: cuestionario abierto \\
\hline $\begin{array}{l}\text { 1. ¿Cómo funcionan los planes comprehensivos? ¿Son realmente } \\
\text { planes integrales de ordenación? }\end{array}$ \\
\hline 2. ¿Qué papel representan las comisiones de planificación territorial? \\
\hline $\begin{array}{l}\text { 3. ¿Cómo funcionan o qué mecanismos se emplean en la cooperación } \\
\text { y coordinación interadministrativa en materia de planificación } \\
\text { comprehensiva? }\end{array}$ \\
\hline $\begin{array}{l}\text { 4. ¿Ha sido relevante la participación ciudadana para la planificación } \\
\text { comprehensiva? }\end{array}$ \\
\hline 5. ¿Cómo ha afectado la crisis económica en esta materia? \\
\hline
\end{tabular}

Fuente: elaboración propia

\section{Planificación comprehensiva en Estados Unidos}

\subsection{Definición de la planificación comprehensiva en Estados Unidos}

La planificación comprehensiva en Estados Unidos (comprehensive planning o urban comprehensive planning) es un procedimiento de ordenación física de un territorio de carácter integral y global, literalmente comprehensivo, con el fin de alcanzar unos objetivos de desarrollo territorial sostenibles. Sus instrumentos de implementación algunas veces también pueden ser conocidos como planes integrales (comprehensive plans), planes maestros (master plans), planes generales (general plans), planes regionales (regional area plans) o también por planes territoriales del gobierno local (local government plans). En Estados Unidos, se entienden como gobiernos locales también las administraciones de los Condados, equivalentes a las delimitaciones administrativas comarcales europeas, y que abarcan superficies similares a las Comunidades Autónomas españolas. De acuerdo con la Asociación Americana de Planificación (American Planning Association, APA) un plan comprehensivo de escala local o de escala supralocal implica la adopción de un reglamento por parte de un cuerpo legislativo de gobierno. Además, este documento debe plasmar, a través de palabras, mapas, ilustraciones o tablas, sus objetivos, políticas y líneas estratégicas para dirigir el desarrollo presente y futuro, social, económico y físico 
(ambiental), del territorio al que da cobertura. Esto incluye un diseño del modelo de desarrollo, tanto para el sector público como para el desarrollo privado, en relación con el uso y aprovechamientos de los usos del suelo (APA, 2002). En gran parte de Estados Unidos se trata de la única política que considera en sus instrumentos de implementación múltiples programas sectoriales para el desarrollo de actividades sobre la totalidad del área planificada, sea esta pública o privada (Kelly, 2010).

Aunque este artículo se centra en el caso de los planes comprehensivos de Arizona y concretamente del condado de Pima y de la aglomeración urbana de la ciudad de Tucson, resulta conveniente destacar que varios trabajos han tratado recientemente todas estas y otras problemáticas sobre la planificación comprehensiva americana, prestando especial interés en los aspectos participativos y comunicativos de la misma (Dandekar \& Main, 2014; Ellin, 2010; Forester, 2013; Mohammadreza, Mohammadi, \& Zangiabadi, 2015; Paris, 2013; Widener, 2013).

\subsection{Origen de la planificación comprehensiva en Estados Unidos: la ciudad en la base}

Uno de los elementos básicos para entender la planificación territorial en Estados Unidos se remonta a los años posteriores a la Revolución Americana de finales del siglo XVIII, donde los derechos relacionados con la propiedad privada personal alcanzan su máxima expresión ${ }^{1}$. Puede servir de ejemplo la máxima en relación con el agua en el oeste americano de first in time, first in right. Lo que se traduce como que la primera persona en aprovechar un recurso obtiene sus privilegios, es decir, se convierte en propietario. Es por ello que las autoridades locales han tenido históricamente pocas opciones de dirigir el desarrollo territorial y urbano en esta época donde la propiedad privada es uno de sus derechos más respetados. Como consecuencia de ello, hasta mediados del siglo XIX no se puede encontrar una intervención sistemática contundente que pueda modificar el devenir urbanístico tan orgánico y personalizado. Esta intervención llegó amparada desde el movimiento de reforma sanitaria anglosajona de 1840 (Sanitary Reform Movement) que relacionó directamente el medio ambiente con el saneamiento urbano. En este momento se consideró primordial la buena localización y la necesidad de grandes espacios abiertos en las ciudades, como por ejemplo parques, con el objetivo último del saneamiento y también del entretenimiento. El ejemplo paradigmático es el parque diseñado por Frederick Law Olmsted de Central Park en Nueva York (Campbell \& Fainstein, 2012). El incremento de la urbanización industrial de finales del siglo XIX y de principios del siglo XX en las grandes urbes americanas supuso que tuviera igualmente un gran impacto el Movimiento norteamericano de embellecimiento de la ciudad (City Beautiful Movement) con el objetivo de paliar los problemas y la insalubridad de la industrialización urbana. Posteriormente, liderado por el británico Ebenezer Howard (1898 y 1902), el movimiento

1 Como se recoge en la Enmienda $\vee$ de la Carta de Derechos de los Estados Unidos de América de 1791 (ver Hallaq, 2014). 
urbanístico de la Ciudad Jardín (Garden City Movement) también irrumpió con fuerza en Estados Unidos y conllevaría la aparición de una necesaria planificación territorial de carácter integral. El primer auténtico plan comprehensivo fue elaborado por Daniel $\mathrm{H}$. Burnham para la gran ciudad de Chicago en 1909, reconocido no solamente por ser el primero de su clase sino también por su gran funcionalidad. Otras ciudades como Washington D.C., San Francisco o Cleveland serían también pioneras (Smith, 2006).

Así pues, los orígenes de la planificación territorial y urbana estadounidense se constituyen con las bases legales de la protección de la salud y bienestar de los ciudadanos del siglo XIX. Se trata de una competencia de escala local que deriva de la legislación estatal. Sin embargo, en la mayoría de estados los planes comprehensivos no son requeridos por ley. La normativa federal americana ofrece un marco fundamental con el desarrollo de dos documentos legales elaborados en el primer tercio del siglo XX por el Departamento de Comercio de Estados Unidos. El primero de ellos es el proyecto de la Standard State Zonning Enabling Act de 1926 que no pasó nunca más allá del Congreso pero, debido a la necesidad planificadora, muchos estados la adoptaron como marco para institucionalizar la planificación comprehensiva. En consecuencia, tras ese primer documento frustrado, una nueva legislación surgió del mismo Departamento de Comercio, dando lugar a la Standard City Planning Enabling Act de 1928. Esto demuestra el gran interés en esta materia por parte del Gobierno de Estados Unidos y la insuficiencia legal sobre la misma para los estados del país por aquellas fechas. Además, el propio Gobierno de los Estados Unidos apoyaría a las administraciones locales con la iniciativa de elaborar una planificación comprehensiva en muchos estados donde no se exigían legalmente este tipo de planificaciones comprehensivas (Cullingworth \& Caves, 2013). Para un mejor análisis de la planificación territorial americana desde el punto de vista de los derechos de propiedad del suelo, se sugiere ver otros trabajos (Needham, 2006; Needham \& Hartmann, 2016), ya que este no es el cometido de este trabajo.

Por otra parte, también se produjo un desarrollo profesional en 1967 de la figura del planificador gracias a la conocida como "Enmienda de Wetmore (Louis B.)", en la que el Instituto de Planificadores Americanos añade a su declaración de propósitos de 1938 la planificación comprehensiva y la regulación de los usos del suelo, y en la que también se da cabida dentro del instituto profesional tanto a la figura del planificador físico como del planificador social (Kelly \& Becker, 2000).

Sin duda la publicación de otros importantes trabajos tuvo una gran relevancia en la evolución de la planificación territorial estadounidense e internacional. Este es el caso de la obra de lan L. McHarg titulada Design with Nature (1969), a finales de la década de los sesenta, que sería un hito internacional de gran relevancia presentando un nuevo punto de vista al fusionar los procesos de planificación con las características propias del medio ambiente natural. Pocos años antes Lewis Mumford con su libro The City in History: Its Origins, Its Transformations, and Its Prospects (1961) 
abordaría de forma crítica el desarrollo urbano haciendo hincapié en la problemática de la urbanización dispersa (urban spraw) y los consecuentes problemas sociales en parte derivados de la estructura de las ciudades occidentales modernas y cómo la planificación debe hacer énfasis en la relación entre la población y el medio que habitan. En esta misma línea se acuñó el concepto de "megalópolis" (Gottmann, 1961) haciendo alusión a la vasta franja urbanizada noroccidental de Estados Unidos. Desde entonces, el urban sprawl se ha convertido en un tema de estudio importante en Estados Unidos, y en el mundo, en relación con el crecimiento, el consumo de suelo y la pérdida de espacios libres (Zinn, 2004).

Uno de los movimientos, de corte arquitectónico, que trata la problemática del urban sprawl americano es el Nuevo Urbanismo (New Urbanism). Por su parte, la formación de un Congreso para el Nuevo Urbanismo a modo de organización no gubernamental, asume la necesidad de la cohesión social y la responsabilidad de las decisiones tomadas en el proceso de diseño urbano. Sus miembros adoptaron una carta de principios en 1996 en la que se establece la necesidad de restauración de los centros urbanos siendo coherentes con las relaciones existentes en las regiones metropolitanas y reconfigurando el fenómeno del urban sprawl en las periferias urbanas (Leccese \& McCormick, 2000). En esta línea se podría incluir el citado concepto de "postmetropolis" de Edward Soja $(2008 ;$ 2011), donde resulta necesario considerar un modelo basado en las regiones y regiones urbanas más que en las metrópolis para hacer frente a los continuos procesos de urbanización. Soja aborda el proceso de transformación de la postmetrópolis desde una perspectiva esencialmente anglosajona y referida al espacio urbano norteamericano. El autor utiliza este concepto para englobar todos los cambios que afectan a las ciudades en las últimas décadas del siglo XX, así como también los de inicios del siglo XXI con la crisis económica financiera global. Como ejemplo de cambio se pueden reconocer la pérdida creciente de población en los centros de las ciudades de todo el mundo, o que los límites entre lo rural y urbano son cada vez más dinámicos por lo que requieren un modelo con visión regional.

Los primeros planificadores en Estados Unidos, que fundamentalmente eran ingenieros, arquitectos, urbanistas y abogados, estaban de acuerdo en la necesidad de un instrumento de ordenación del territorio o plan territorial comprehensivo de carácter público y de largo recorrido para el desarrollo de las ciudades. Se ha demostrado que planificar de forma comprehensiva refuerza las relaciones entre los elementos funcionales de un plan a lo largo del tiempo y del espacio (Kelly, 2010). Merece la pena destacar que los planes comprehensivos no surgen como un mero recurso técnico, en origen personificado en grandes planificadores, como Burnham en el Plan de Chicago de 1909, sino que también es una herramienta para implicar a la sociedad en el proceso de desarrollo y ordenación territorial, aunque los primeros planes solamente contaban con determinados miembros o élites en la toma de decisiones (Kelly, 2010). Siguiendo al mismo autor, los tres factores importantes que se incluyen en un plan comprehensivo son: 1) tener en cuenta la totalidad del 
territorio jurisdiccional a planificar; 2) incluir todas las materias relacionadas con el desarrollo físicoterritorial de la comunidad a ordenar; y, por último, 3) avanzar un horizonte del plan de largo plazo. Lo cierto es que existen numerosas publicaciones sobre planificación comprehensiva en Estados Unidos pero no es fácil encontrar manuales que detallen los contenidos previstos para sus instrumentos e implementación. Podemos mencionar que los manuales generales más destacados con anterioridad al siglo XXI se reducen a cinco documentos que se dedican a tratar los contenidos elementales (Bassett, 1938, 1940; Kelly \& Becker, 2000; Kent, 1964; Anderson, 1995), pero que se pueden considerar desfasados a excepción de la reciente actualización de Eric D. Kelly (2010).

Como se ha dicho, estos planes comprehensivos surgen en la mayor parte de Estados Unidos como los únicos que consideran múltiples programas y actividades en el territorio a ordenar siendo este de propiedad pública o privada. Este detalle es bastante significativo si tenemos en cuenta la historia relativa al sistema de propiedad privada que ha regido en Estados Unidos como ya se ha comentado.

\subsection{Marco administrativo y normativo de la planificación comprehensiva en Estados Unidos}

La normativa de la planificación comprehensiva está directamente ligada a los distintos niveles con competencia administrativa. Es importante saber que existen dos tipos de gobierno fundamentales que dominan la planificación y control de los usos del suelo en Estados Unidos: las entidades locales, formadas por ciudades y pueblos (cities and towns), y los condados (counties). Sin embargo, también podemos encontrar otra unidad administrativa municipal (los townships) que hacen referencia a parcelas de 6 millas cuadradas $\left(15,54 \mathrm{~km}^{2}\right)$, que a su vez se dividen en 36 secciones y que sirven como mapa de referencia para desarrollar tareas locales de detalle pero, en ocasiones, también para la planificación y zonificación de usos del suelo (Kelly y Becker, 2000; Kelly, 2010). Aunque la planificación territorial suele ser competencia de estas administraciones, los estados también pueden formular un plan comprehensivo de carácter estatal, como ocurre en el caso de Oregón con sus antiguos documentos sobre Statewide Goals \& Guidelines y el nuevo Plan estratégico estatal de 2014, que constituye un marco de referencia para la planificación comprehensiva $^{2}$. Por otra parte, aunque no exista una competencia federal directa en esta materia, la planificación comprehensiva sí que se ve afectada por legislaciones de carácter sectorial a nivel federal. Un ejemplo de normativa sectorial que afecta a la planificación comprehensiva en Estados Unidos son las revisiones sobre conservación ambiental obligatorias establecidas por el Congreso Federal para los Planes Generales Comprehensivos de Gestión de los Parques Nacionales americanos.

2 Para ver un inventario de más ajustado de estados relacionados con su estilo de planificación comprehensiva a comienzos del siglo XXI se recomienda ver el trabajo de APA (2002). 
Para entender el contenido especificado por la normativa de planificación comprehensiva en Estados Unidos deben consultarse primeramente las bases establecidas en los documentos del primer tercio del siglo XX. Una vez dicho esto, y atendiendo a la histórica Standard State Zonning Enabling Act de 1926, las decisiones sobre usos del suelo deben realizarse de forma coherente con los planes comprehensivos, con lo que en principio se garantizaría la democracia y la racionalidad de las medidas tomadas (Kelly \& Becker, 2000). En la Sección Primera de este documento legal de 1926 se estableció el deber de garantizar la salud, la seguridad, los valores y el bienestar general de la comunidad otorgando el poder a las administraciones locales para regular y restringir actividades urbanísticas. En la Sección Segunda y Sección Tercera se establece la necesidad de dividir en distritos esas regulaciones y se remarca la necesaria coherencia con un plan integrador, como es el propio plan comprehensivo.

Por otra parte, la Standard City Planning Enabling Act de 1928, que sí entró en vigor, se centra en autorizar la creación de un Plan Maestro y de una comisión de planificación para las distintas administraciones públicas locales (ciudades y pueblos) o regionales (condados). Esta nueva legislación profundiza en conceptos, contenidos y procedimientos que deben llevar a cabo las comisiones de elaboración de los planes, entre los que podemos destacar la necesidad de recabar información a través de estudios y encuestas integrales para conocer las condiciones presentes y de futuro sobre el crecimiento de los municipios. Además, en esta ley se encuentran los elementos destinados a la democratización y transparencia de la planificación como demuestra el requerimiento de establecer audiencias públicas previas a la aprobación de un plan comprehensivo, así como para sus modificaciones, ampliaciones o renovaciones.

El conjunto normativo de la planificación comprehensiva en Estados Unidos fue ampliado con el programa de planificación 701 del Departamento de Vivienda y Desarrollo Urbano de la Ley de Vivienda de $1954^{3}$. En él se aprecian los fundamentos originales de la planificación comprehensiva centrados en la planificación física de usos del suelo (land use planning). Además, también encontramos el programa 701 de Gestión de Aguas Residuales de la Ley de Control de Contaminación de Aguas Federal de $1948^{4}$ derivada de la Agencia de Protección del Medio Ambiente americana. Ambos programas reforzaron la visión a largo plazo de la planificación comprehensiva, así como su delimitación territorial y el énfasis en la planificación física ambiental. Además, durante los años sesenta y principios de los setenta, la planificación territorial fue enriqueciéndose en Estados Unidos gracias al desarrollo legislativo sectorial federal. Puede servir como ejemplo la National Historic Preservation Act de 1966, la Coastal Zone Management Act de

3 Fondos para la planificación comprehensiva bajo la sección 701 (Housing Act of 1954).

4 La base de la Ley de Agua Limpia se promulgó en 1948 y se llamó Ley Federal de Control de la Contaminación del Agua (Clean Water Act 1977 SEC. 208 [33 U.S.C. 1288] Areawide Waste Treatment Management). 
1972 o el requerimiento de evaluar el estado e impacto ambiental de planes, programas y proyectos con la National Environment Policy Act de 1969. Finalmente, el conjunto normativo presentado, concreta en la planificación comprehensiva el objeto de planificar los usos del suelo, el transporte, la vivienda, el medio ambiente, los parques y espacios abiertos, la infraestructura educativa, la igualdad social, y la preservación de barrios históricos.

Tras el desarrollo de las mencionadas normas sectoriales estatales con incidencia en la planificación comprehensiva hasta los años 80 , surgieron posteriormente nuevas legislaciones relacionadas con la teoría urbanística del crecimiento inteligente de las ciudades (smart growth) en numerosos estados del país. En el siguiente bloque se desarrolla ampliamente cómo este fenómeno afectó directamente en el estado de Arizona y cómo estas influencias teóricas y normativas han influenciado la planificación territorial comprehensiva y concretamente los contenidos de los planes comprehensivos.

\subsection{Planificación territorial en Estados Unidos y la conceptualización europea}

Es conocida por los expertos una amplia tradición localista en la planificación territorial de los países británicos (Davoudi \& Madanipour, 2015; Haughton \& Allmendinger, 2013). Esta tradición localista anglosajona se reconoce también en Norteamérica. Su hito más significativo es la aprobación del plan local mediante referéndum. Esta democratización no sólo existe en Europa, también se da en Estados Unidos. A diferencia de la orientación económica de la ordenación del territorio francesa o de la expansión de la doctrina de planificación física del territorio bajo el punto de vista del acuerdo en Europa (ver Faludi, 2010), en Estados Unidos existe un modelo más colaborativo y comunicativo de planificación territorial. Este modo de planificación tiene una cierta tradición en el continente americano y es defendido por el ya citado movimiento del Nuevo Urbanismo. En esta línea, Emily Talen (2005) remarca la importancia de los modelos de planificación tradicionales, puesto que si bien suponen un modo de planificación desde arriba (top-down) estas propuestas sirven como marco o escenario inicial para el debate o la discusión de forma ordenada sobre la complejidad del territorio o de las ciudades actuales por parte de todos los actores territoriales implicados. Desde comienzos de este siglo numerosos trabajos continúan ratificando la conveniencia de la planificación colaborativa basada en escenarios (scenario planning) frente a un contexto ambiental y territorial actual de gran incertidumbre (Peterson, Cumming \& Carpenter, 2003). Lo cierto es que muchos actores territoriales (stakeholders), no están tan acostumbrados a trabajar con modelización espacial pero sí que están familiarizados con el trabajo a través de escenarios (Petrov, Shahumyan, Williams \& Convery, 2011). Poner a trabajar juntos a científicos, otros actores territoriales y a los tomadores de decisiones, no solo en la fase de creación de escenarios posibles sino que también en la aplicación práctica del modelo espacial, supone que se puedan compartir experiencias y aprender entre todos ellos, lo que debe conllevar necesariamente a una mejor y más efectiva planificación territorial sostenible (Petrov et al., 2011). 
Desde comienzos del siglo XXI, Susan Fainstein (2000) y otros autores americanos también reconocen la necesidad de superar las brechas existentes entre los tomadores de decisiones, los científicos, los actores territoriales y la ciudadanía en general. Estos problemas no son menores en el caso europeo (Stead, 2013). Por otro lado, en los modelos de planificación más comunicativos y colaborativos, el planificador toma el papel de mediador entre todos estos actores. Un ejemplo de enfoque colaborativo es el llevado a cabo por el Instituto de Recursos Hídricos del Cuerpo de Ingenieros del ejército de Estados Unidos ${ }^{5}$. Este enfoque es reconocido bajo el programa de Planificación con una visión compartida (Shared Visión Planning). En él se combinan los métodos de la planificación tradicional, la participación ciudadana de forma estructurada y la elaboración de modelos informáticos. El objetivo principal de este enfoque es mejorar en relación con la toma de decisiones y el desarrollo sostenible al facilitar un entendimiento común de los problemas entre los distintos actores territoriales implicados, a través de modelos informáticos o escenarios adaptables a los cambios que resulten y comprensibles para todos (Cardwell, Langsdale \& Stephenson, 2009).

El planteamiento de un objetivo común como una utopía es en ocasiones la circunstancia más discutida en estas formas de planificación basadas en un modelo colaborativo y más comunicativo, en el que generalmente se debe alcanzar un consenso. Recientemente Charles Hoch (2016) plantea la integración de esta utopía con la proposición de un modelo basado en escenarios para elaborar un instrumento de planificación. Según este autor, la utopía describe el futuro deseable o ideal, mientras que el planteamiento de posibles escenarios permite comparar las mejores alternativas y mostrar las narrativas y explicaciones detrás de cada una de las posturas y actores territoriales implicados. Como consecuencia, el proceso de planificación se vería notablemente enriquecido y al menos se convertiría en un camino de aprendizaje mutuo para los distintos actores territoriales implicados.

En relación con la experiencia de planificación colaborativa en el contexto occidental, se constata que el papel de la conformación de redes de conocimiento supone un aumento del grado de complejidad, y que son fundamentales para resolver la incertidumbre creciente en el proceso de planificación territorial. Algunos autores, matizan que las soluciones territoriales propuestas no deben ser solo técnicas, sino que necesitan colaboración y participación de los interesados (Espluga \& Subirats, 2007; Hernández-Mora \& Ballester, 2011; Lauber, Decker \& Knuth, 2008; Pahl-Wostl, 2007). Para ello se requiere un modelo de comunicación de doble vía en el que los participantes no sean meramente informados sino que también aporten (Gao, Kornov \& Christensen, 2013; O’Toole, Wallis \& Mitchell, 2009). Por tanto, esto supondría el uso de más de un medio de comunicación y asumir que existirán pérdidas de entendimiento. Asimismo, ante la

5 Institute for Water Resources. Shared Vision Planning Program (US Army Corps of Engineers) http://www.iwr.usace. army.mil/, http://www.sharedvisionplanning.us/ 
complejidad de los actores implicados es importante tratar las relaciones de confianza o desconfianza y conocer la distribución de poder e intereses (O’Toole et al., 2009).

A continuación se profundiza en el caso de Arizona y la experiencia planificadora en el condado de Pima, de lo que se pueden extraer algunas consideraciones y aportaciones también de gran interés.

\section{Crecimiento inteligente y planificación comprehensiva en el estado de Arizona}

\subsection{Impacto del crecimiento inteligente en la planificación comprehensiva}

En el estado de Arizona, desde 1973 bajo el amparo de los Estatutos de Arizona (Arizona Revised Statutes, ARS), se ha requerido a la mayoría de ciudades, pueblos y condados el desarrollo de planes comprehensivos que ordenen de forma integrada sus usos del suelo, el tráfico rodado, viviendas, servicios públicos e instalaciones, así como la conservación, rehabilitación y reconstrucción de sus territorios entre otros elementos estructurantes. Este impulso a la planificación de carácter integral se vio notablemente fortalecido gracias a las preocupaciones derivadas de la teoría urbanística conocida como crecimiento inteligente o Smart Growth. En Arizona se ha estado trabajando activamente para gestionar el crecimiento inteligente de las ciudades y preservar los espacios abiertos del estado durante más de una década.

De acuerdo con el espacio web oficial de la Oficina de Arizona sobre Crecimiento Inteligente ${ }^{6}$, debido a que las tasas de crecimiento real de la población aumentaron significativamente en la década de 1990 en muchas ciudades americanas, surgió un movimiento sociopolítico general para ofrecer soluciones a la hora de gestionar este crecimiento tan acelerado. En el caso de Arizona, en el año 1998, a través de una coordinación entre el poder legislativo del estado de Arizona, los ciudadanos interesados y la Oficina del Gobernador de Arizona, se llevó a cabo un esfuerzo integral para abordar los temas relacionados con el crecimiento urbano que conllevaron la aprobación de la Growing Smarter Act del año 1998. Esta ley fortaleció los elementos de planificación y añadió cuatro nuevos contenidos a tratar por la planificación territorial: los espacios abiertos, las nuevas áreas de crecimiento, la planificación ambiental moderna y el coste de desarrollo. Además, la Growing Smarter Plus Act del año 2000 profundizó en estos aspectos y en los relacionados con la planificación de los usos del suelo en Arizona.

Este conjunto legislativo, conocido en Arizona como growing smarter legislation, modifica la planificación comprehensiva de usos del suelo y la zonificación urbana, incluyendo la adquisición de espacios abiertos, dando a los condados, ciudades, pueblos y ciudadanos en general una serie de herramientas y restricciones para gestionar su crecimiento. Uno de estos derechos es el de votar

6 Ver hittp://old.azcommerce.com/SmartGrowth/ 
en referéndum la aprobación de los planes comprehensivos. Estas premisas del Smart Growth son recogidas por los Estatutos de Arizona (ARS §9-461.05 y ARS §11-821). Además, esto ofrece un cuerpo legal sólido para declarar y preservar espacios abiertos más allá de lo previsto por el Departamento del Estado sobre esta materia. Su principal objetivo es que el futuro desarrollo estatal se produzca de una manera más racional, eficiente y sensible con el medio ambiente y que favorezca los intereses de los ciudadanos mediante la promoción de la protección de su patrimonio natural en equilibrio con su competitividad económica. Entre las nuevas características y requisitos que se incorporan a la planificación comprehensiva tras la entrada en vigor de la growing smarter legislation en Arizona, destacan una serie de aspectos fundamentales a la hora de garantizar una planificación territorial inteligente y de calidad ${ }^{7}$ :

1. Se requiere la aprobación del plan por los votantes en las grandes ciudades y las de mayor crecimiento al menos una vez cada diez años. Además, estos planes deben contemplar los recursos hídricos como elemento fundamental.

2. Exige la coherencia de la zonificación de usos con los planes comprehensivos.

3. Exige una participación pública más eficaz en el proceso de planificación.

4. Exige coordinación y cooperación entre las distintas administraciones locales y también con las agencias de planificación regionales.

5. Requiere transparencia en relación con los propietarios del suelo y la carencia de servicios e instalaciones disponibles.

6. Requiere el permiso de los propietarios del suelo para reclasificar un terreno en espacio abierto.

7. Autoriza a las administraciones locales para designar los límites de áreas de servicio.

8. Permite a los condados imponer cuotas o tasas de desarrollo de acuerdo a los estatutos.

9. Permite a las ciudades incentivar desarrollos o planes en distritos.

De estas exigencias se pueden destacar el refuerzo de la participación ciudadana, una mayor transparencia, así como la coordinación y cooperación institucional, entre otros. Todos estos son aspectos fundamentales para garantizar una planificación territorial inteligente y de calidad.

Por otra parte, resulta oportuno destacar que, como complemento a la orientación fuertemente urbana de esta normativa, surgió el programa Growing Smarter Planning Grant con el propósito de ayudar, generalmente a través de subvenciones, a las comunidades rurales y condados en el cumplimiento de su planificación para respetar los nuevos requisitos. Sólo se tendrían en cuenta

7 Adaptado de Arizona Office of Smart Grow, Arizona Commerce Authority. Retrieved from https://azcommerce.com/ 
aquellas comunidades rurales o condados que se comprometieran en la elaboración de un plan comprehensivo. Estas ayudas apoyan el desarrollo de la investigación, el análisis y la preparación de los mapas y gráficos pertinentes de los planes comprehensivos, así como la participación ciudadana y la implementación de los planes mediante la actualización de la ordenanza de zonificación u otros planes específicos.

Por otro lado, la growing smarter legislation da pie a la elaboración, por parte del Estado de Arizona, de los Planes de usos del suelo conceptuales (Conceptual State Land Use plans), aunque todavía no están operativos en Arizona. Se trata de unos planes territoriales conceptuales de carácter integral para grandes espacios abiertos (Urban State Trust Lands), y que básicamente se asociarían a terrenos o espacios abiertos a menos de una milla de las ciudades o pueblos menores de 250000 habitantes y de tres millas para el caso de ciudades superiores a esta cifra. Estos espacios están regulados por los ARS (ARS, 37-331 Urban Lands Development) en tanto que planes generales del estado de Arizona. También, se definen sus contenidos en este mismo texto legal y se formula la obligación de formar un comité estatal para elaborarlos y revisarlos periódicamente, al menos cada diez años, entre otras cuestiones. Además, esta normativa otorga competencias al estado para declarar los usos e intereses sobre estos terrenos. Generalmente, siempre que no existiera un plan comprehensivo, estos planes de usos del suelo conceptuales elaborados por la comisión estatal podrían designar estos espacios como apropiados para su desarrollo urbanístico o bien como recursos a conservar si fuera necesario. La legislación establece criterios en coherencia con la Growing Smarter Legislation para evitar la dispersión urbana y favorecer el interés general y la planificación a largo plazo. Por tanto, los usos del suelo adecuados para estos terrenos (comercial, agrícola, industrial, residencial, espacios abiertos o uso recreativo) podrían ser preestablecidos. Además, se pueden determinar restricciones en espacios con valor ambiental o riesgos naturales, así como tomar medidas preventivas en espacios idóneos para el desarrollo de otros elementos e infraestructuras de interés general. Estos planes estatales deben ser consultados por las administraciones locales y regionales además de por las distintas agencias de planificación; también deben ser consistentes con el resto de políticas y planes de las administraciones locales y regionales. Sin embargo, al no estar operativos estos planes de competencia estatal, debe remarcarse que no existe un marco de referencia para los planes comprehensivos regionales y locales en Arizona.

\subsection{Contenidos y características básicas de los planes comprehensivos}

La tabla siguiente (Tabla 2) refleja los contenidos y características básicas del estado de Arizona en relación con las normas para los planes comprehensivos en Estados Unidos de principios del siglo XX (APA, 2002), la existencia y obligatoriedad o no de la planificación estatal, local y municipal, así como los elementos contenidos en los propios planes comprehensivos. 
Tabla 2. Requisitos normativos para los planes comprehensivos en el estado de Arizona

\begin{tabular}{|c|c|c|}
\hline $\begin{array}{l}\text { Similitud con las } \\
\text { normativas de } 1926 \text { y } \\
1928\end{array}$ & \multicolumn{2}{|c|}{$\begin{array}{l}\text { Moderadamente actualizado (muchos } \\
\text { cambios significativos pero todavía se } \\
\text { asemeja a las leyes de 1928) }\end{array}$} \\
\hline $\begin{array}{l}\text { Obligatoriedad del plan } \\
\text { comprehensivo por el } \\
\text { municipio }\end{array}$ & \multicolumn{2}{|c|}{$\begin{array}{l}\text { La elaboración del plan por el municipio es } \\
\text { obligatoria si se cumple con la condición de } \\
\text { crear una comisión de planificación } \\
\text { (ciudades y pueblos). } \\
\text { Obligatorio para los Condados }\end{array}$} \\
\hline $\begin{array}{l}\text { Política de usos del suelo } \\
\text { Estatal }\end{array}$ & \multicolumn{2}{|c|}{$\begin{array}{l}\text { No ( con la excepción de los inoperativos } \\
\text { planes de usos del suelo estatales) }\end{array}$} \\
\hline Peso del estado & \multicolumn{2}{|l|}{ Débil } \\
\hline $\begin{array}{l}\text { Estatutos del estado de } \\
\text { Arizona }\end{array}$ & \multicolumn{2}{|c|}{$\begin{array}{l}\text { A.R.S. Cities \& Towns 9-461 ; A.R.S. } \\
\text { Counties 11-801 }\end{array}$} \\
\hline \multicolumn{3}{|c|}{ Contenidos de los planes comprehensivos } \\
\hline \multicolumn{2}{|c|}{$\begin{array}{c}\text { Contenidos de los planes } \\
\text { comprehensivos: } \\
\text { Usos del suelo (2) } \\
\text { Vivienda (2) } \\
\text { Agricultura, bosques y espacios } \\
\text { abiertos (3) } \\
\text { Riesgos naturales (1) } \\
\text { Reurbanización (1) } \\
\text { Entretenimiento y ocio (2) } \\
\text { Energía (1) } \\
\text { Calidad del aire (1) } \\
\text { Transporte (2) } \\
\text { Servicios comunitarios (1) } \\
\text { Políticas y Planes de Acción (2) } \\
\text { Participación pública (2) } \\
\text { Otros planes específicos (3) }\end{array}$} & $\begin{array}{l}\text { Otros elementos no incluidos: } \\
\text { Desarrollo económico } \\
\text { Límites del crecimiento urbano } \\
\text { Áreas críticas y sensibles } \\
\text { Servicios sociales } \\
\text { Diseño comunitario } \\
\text { Preservación histórica } \\
\text { Implementación } \\
\text { Coordinación local } \\
\text { Otros elementos }\end{array}$ \\
\hline
\end{tabular}

Fuente: elaboración propia a partir

de la Asociación Americana de Planificación (APA, 2002)

A principios del siglo XXI, la planificación comprehensiva de Arizona presenta escasa presencia estatal. Por otra parte, los planes comprehensivos existentes guardan estrecha relación con las legislaciones del primer tercio del siglo pasado. Además, en ellos destacan los contenidos dedicados a elementos de detalle desarrollados para la ordenación de los usos de los suelos agrícolas, masas forestales y espacios abiertos. Merece la pena mencionar la poca atención prestada por los planes a los contenidos de desarrollo económico y a la propia implementación del plan. Asimismo, resulta evidente la relación de los planes comprehensivos de Arizona con un modelo de planificación física y de control de los usos del suelo en el que las premisas de la Growing Smarter Legislation de Arizona todavía no han calado profundamente en los planes 
aprobados antes de 2002 examinados por el trabajo de la APA (2002). La prueba más clara de ello es que, aunque la participación ciudadana sí es considerada como elemento clave en los planes, otros elementos como la coordinación y cooperación institucional o la propia implementación de las políticas del plan no resultan relevantes (ver Tabla 2). Esto implica que los planes comprehensivos sean primordialmente de carácter estratégico, al no contener mecanismos legales propios para alcanzar los objetivos e implementar las políticas de actuación propuestas.

Desde el punto de vista legal, existen dos capítulos específicos en los Estatutos de Arizona que contemplan las dos categorías fundamentales de planes comprehensivos. El Capítulo 6 del Título 11 de los ARS sobre planificación y zonificación de condados; y el Capítulo 4 del Título 9 de los ARS dedicado a las competencias generales en planificación comprehensiva y sectorial de ciudades y pueblos de Arizona.

De acuerdo con los Estatutos (Title 11 Counties - Chapter 6 County Planning and Zoning), se establece la creación de una comisión de planificación y zonificación encargada de desarrollar y mantener un plan comprehensivo de amplio horizonte para el total de la jurisdicción de los condados de forma coherente con la planificación estatal. Los Estatutos también desarrollan ampliamente los contenidos y objetivos de esta planificación y determinan que los estudios y recomendaciones de dicho plan deben incluir una serie de elementos fundamentales que se ven ampliados de acuerdo con el tamaño poblacional de cada condado (Tabla 3).

\section{Tabla 3. Elementos requeridos para los planes comprehensivos de los condados de Arizona}

Elementos comunes: infraestructura de transporte y comunicaciones, incluyendo la bicicleta, así como puentes, edificios públicos, servicios públicos, escuelas, parques, espacios libres, calidad, acceso y variedad de viviendas,

aparcamientos, caminos y senderos, espacios forestales, áreas naturales, presas, conservación de recursos naturales, calidad del aire y calidad del agua y riesgos de inundación.

Para condados de más de 125000 habitantes: usos del suelo, circulación y transporte, planificación energética y planificación hidrológica.

Para condados de más de 200000 habitantes: espacios abiertos, áreas de crecimiento, planificación medioambiental, coste de desarrollo.

Fuente: traducción a partir de los Estatutos de Arizona

(ARS, Title 11 Counties - Chapter 6 County Planning and Zoning)

Para cada uno de los elementos, los Estatutos también incluyen unos contenidos mínimos de forma sintética. Además de todos estos elementos, los planes comprehensivos de los condados deben implantar medidas propias de seguimiento para establecer la necesidad de modificaciones puntuales, adaptaciones significativas del propio plan o la renovación del mismo. Asimismo, este plan debería ser renovado cada diez años. 
Por otra parte, en relación con la planificación de ciudades y pueblos, igualmente según los Estatutos de Arizona (Title 9 Cities and Towns - Chapter 4 General Powers), se establece la necesidad de creación de una agencia, una comisión y un departamento de planificación locales encargados de desarrollar y mantener un plan comprehensivo de amplio horizonte de forma coherente con la planificación estatal. Los contenidos básicos determinados por los Estatutos de los planes para ciudades y pueblos son el establecimiento de una declaración de objetivos comunitarios y de políticas para llevarlos a cabo sobre los siguientes elementos fundamentales, atendiendo al tamaño y crecimiento de cada ciudad (Tabla 4).

\section{Tabla 4. Elementos requeridos para planes comprehensivos de ciudades y pueblos de Arizona}

Elementos comunes: usos del suelo, circulación y transporte.

Para ciudades mayores de 10000 habitantes o con un crecimiento anual superior al $2 \%$ : espacios abiertos, áreas de crecimiento, planificación medioambiental, coste de desarrollo, recursos hídricos.

Para ciudades mayores de 50000 habitantes: conservación ambiental, espacios recreativos, planificación integral del sistema de transporte y comunicaciones, servicios públicos, edificios públicos, vivienda, conservación, rehabilitación y reconstrucción de espacios, seguridad, transporte en bicicleta, energía, preservación y revitalización de barrios.

Fuente: elaboración propia a partir de los Estatutos de Arizona

(ARS, Title 9 Cities and Towns - Chapter 4 General Powers)

Como se puede apreciar en los recuadros anteriores (1 y 2), los contenidos establecidos por los Estatutos de Arizona para los planes regionales y locales son prácticamente idénticos. Además, son coherentes con los principios de la legislación Smart Growth del estado de Arizona.

Figura 1. Instrumentos y normativa de planificación territorial en el estado de Arizona

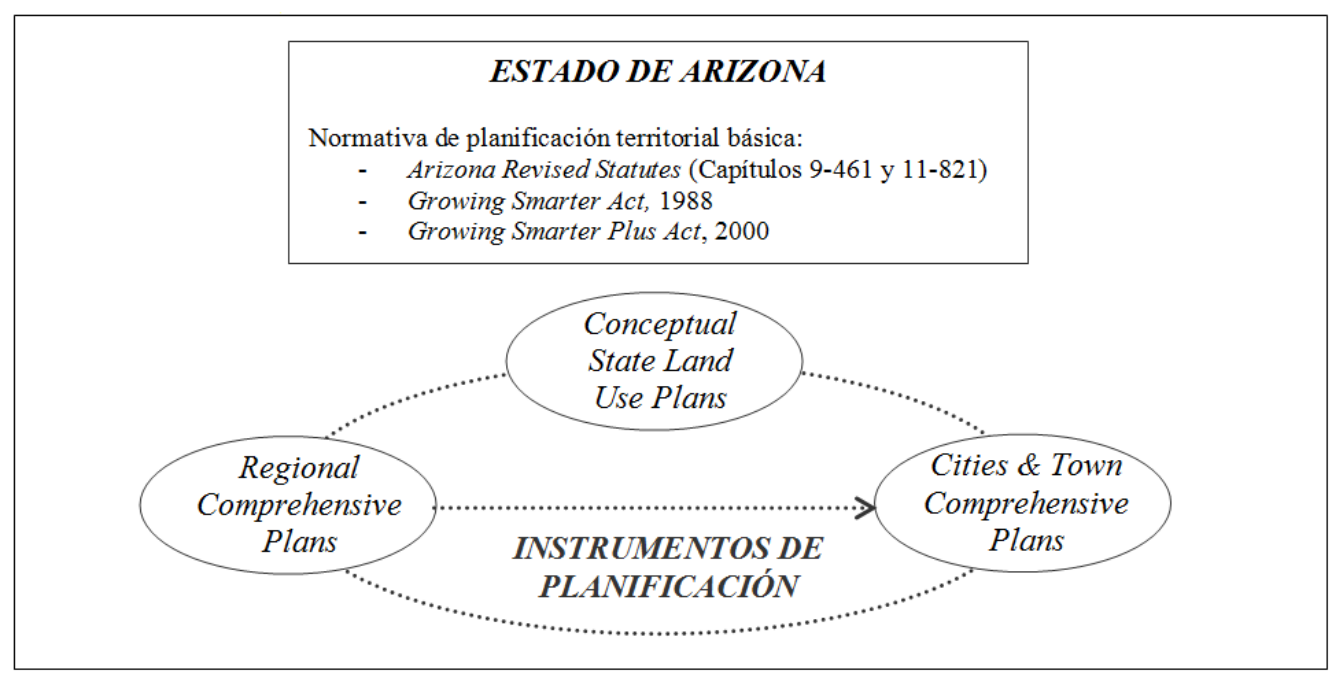

Fuente: elaboración propia

En la figura siguiente (Figura 1), se muestran los principales instrumentos y normas que enmarcan la planificación territorial en el estado de Arizona. En los siguientes epígrafes se profundiza sobre la 
evaluación, el seguimiento, así como sobre la implementación práctica de los planes comprehensivos del condado de Pima.

\subsection{Mecanismos de evaluación y seguimiento basados en la gobernanza y la participación}

En relación con los aspectos relativos a la evaluación y seguimiento de los planes comprehensivos territoriales de Arizona, cabe decir que tanto las directrices establecidas por la legislación estatal, como la normativa Growing Smarter Legislation y los Estatutos de Arizona establecen una renovación de los planes comprehensivos al menos cada 10 años. Como ya se ha mostrado, la Standard City Planning Enabling Act de 1928 determinó los aspectos fundamentales a tener e cuenta para la aprobación, renovación y modificación de los planes, sentando unas bases de democratización y transparencia al exigir audiencias públicas previas a estos procedimientos. Por su parte, los Estatutos de Arizona dedican secciones específicas para estos procedimientos en las que se requiere legalmente la adopción de medidas para la participación pública de forma continua durante todas las fases del proceso de desarrollo y renovación del plan, así como la difusión de las propuestas y alternativas, la presencia de audiencias públicas, la oportunidad de hacer discusión y comentarios, la existencia de programas específicos de comunicación y servicios de información. Además se hace hincapié en las necesarias consultas y la coordinación horizontal y vertical administrativas. Por último, se establece que la aprobación, renovación o modificación substancial de los planes comprehensivos de ciudades y pueblos deben ser ratificadas por al menos dos tercios de los votos del gobierno municipal y, lo más importante, de acuerdo con la subsección 9461.06-M de los Estatutos de Arizona, deben ser aprobados mediante referéndum público por los propios votantes municipales.

Atendiendo a la evaluación y el seguimiento de los planes comprehensivos en Estados Unidos y Canadá, estas evaluaciones estaban tradicionalmente ligadas a las políticas de desarrollo, a las evaluaciones de sostenibilidad o a la revisión de los propios planes (Berke \& Manta, 2000; Seasons, 2003). A comienzos del siglo XXI ya existía gran diversidad de información e indicadores accesibles para la evaluación y el seguimiento; aunque estos recursos no se aprovecharan al máximo en la práctica (Seasons, 2003). Según el mismo autor, y desde un punto de vista crítico, la reducción de fondos desde los años noventa supone una de las principales causas del descenso del presupuesto, del personal y del nivel de apoyo a la inversión para la innovación de la planificación territorial integral, así como para su evaluación y seguimiento. Se observa que en la mayoría de ocasiones, siempre se suele responder primeramente a los problemas inmediatos. Como consecuencia, los mecanismos de evaluación y seguimiento de los planes quedan en ocasiones mermados por su esencial perspectiva a medio o largo plazo. Por otra parte, los indicadores de seguimiento son difíciles de encontrar en los documentos de los planes, y menos aún vinculados a los objetivos o políticas de los planes y programas de actuación. Además, Simons (2003) agrega que haría falta lograr un consenso en los criterios, puesto que los objetivos son muy 
dinámicos y pueden variar a lo largo de la vida de los planes tras cambios técnicos y políticos. Por tanto, el apoyo o liderazgo político resulta fundamental. En ocasiones se manifiesta un rechazo institucional hacia la evaluación y el seguimiento de los planes por la asignación de corresponsabilidades que ello implica. Generalmente, la evaluación y el seguimiento son bien recibidos en organizaciones donde existe interés por la mejora constante en su propio sistema de funcionamiento. La evaluación y el seguimiento de los planes deben ser una obligación más que una necesidad, tanto si los resultados son favorables como si son desfavorables (Seasons, 2003). De este autor se concluye que la evaluación y el seguimiento debe ser introducida paulatinamente en el proceso de planificación territorial, puesto que esta práctica requiere un recorrido de aprendizaje debido a su complejidad y dinamismo. Asimismo, la elección de indicadores debe ser muy cuidadosa debido a que suele implicar responsabilidades que afectan a los intereses esenciales de los distintos actores territoriales implicados. Por ello mismo, las instituciones deben distinguir de forma clara los diagnósticos y mediciones genéricos de una verdadera labor de evaluación y seguimiento continuada en el tiempo.

\subsection{Práctica de la planificación comprehensiva en el condado de Pima y la ciudad de Tucson}

Lo primero que se debe advertir en materia de planificación comprehensiva territorial, tanto en el nuevo documento desarrollado para el condado de Pima, Pima Prospers 2015, como en el de la aglomeración urbana de la ciudad de Tucson, Plan Tucson 2013 (Figura 2), es que existe una amplia tradición planificadora, con planes comprehensivos de tercera generación. A pesar de ello, como se ha mostrado, los planes guardan todavía una estrecha relación con los elementos y recomendaciones de las normativas de principios del siglo XX, así como de las premisas de las legislaciones relacionadas con el crecimiento inteligente en Arizona de los años ochenta y noventa. Por otra parte existen algunas innovaciones interesantes, de acuerdo con las entrevistas realizadas en el departamento responsable de la planificación de la ciudad de Tucson, sobre los elementos del Plan Tucson 2013 que se encuentran mejor integrados que las ediciones anteriores y además en el mismo se ha tratado de concretar al máximo para no banalizar ningún concepto. En el caso del último plan comprehensivo del condado de Pima, Pima Prospers 2015, también se ha avanzado desde la anterior edición de 2001 hacia la integración de elementos. Anteriormente la ordenación física o de usos del suelo tenía el peso más importante dentro del plan. Sin embargo, ahora el documento del plan Pima Prospers 2015 presenta un carácter realmente integrado, aunque todavía la ordenación de usos del suelo sigue siendo uno de los elementos estructurantes, como también los aspectos relacionados con la salud pública puesto que la administración del condado tiene fuertes competencias en esta materia. Igual que en el caso del Plan Tucson 2013, los técnicos entrevistados del Departamento de Vivienda y Desarrollo Comunitario del Condado de Pima (Housing \& Community Development Department) advierten que la complejidad característica de 
este tipo de planes se deja notar sobre todo a la hora de la implementación de las políticas del plan. La ordenación de usos del suelo pormenorizado (rezonning) es el principal mecanismo para la implementación puesto que esta ordenanza local (por ejemplo de la ciudad de Tucson) debe ser coherente con el plan comprehensivo del condado de Pima.

Figura 2. Últimas generaciones de planes comprehensivos del condado de Pima y Ciudad de Tucson

\section{PLANES COMPREHENSIVOS DEL CONDADO DE PIMA Y DE LA CIUDAD DE TUCSON}

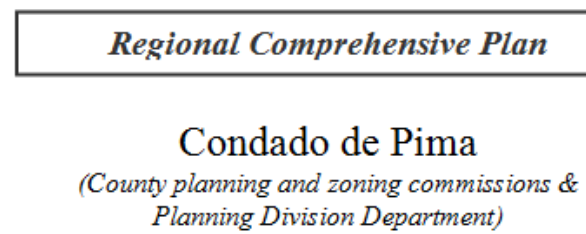

Pima County Comprehensive Plan 2000

Pima Prospers 2015

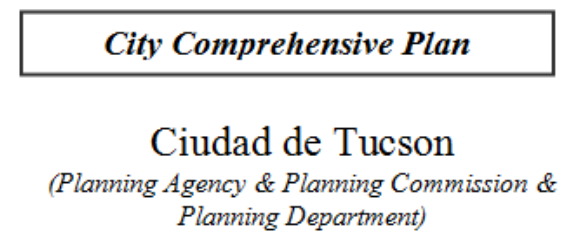

Tucson General Plan 2001

Plan Tucson 2013

\section{Fuente: elaboración propia}

En relación con la jerarquía del sistema de planificación en el condado de Pima, la Junta de Supervisores del condado es la que tiene la última palabra sobre planificación, y únicamente el departamento de planificación del estado de Arizona (State Land Use Department) supedita la labor desarrollada. En realidad la Comisión de Planificación y Zonificación del Condado de Pima revisa los diferentes proyectos de rezonificación de usos y presenta las recomendaciones pertinentes a la Junta de Supervisores que es la que finalmente toma las decisiones. Todo ello no sin dificultades, por las diferentes jurisdicciones afectadas, que se materializan en los conocidos como problemas de ajuste espacial (spatial fit) (Moss, 2012). Además, en relación con la coordinación y cooperación con las administraciones y planificaciones sectoriales, de acuerdo con las respuestas de los técnicos entrevistados en las dos administraciones, se ha tratado la coordinación con todos los departamentos afectados, tanto en la escala local como comarcal, siendo especialmente relevante la colaboración existente con el Departamento de Aguas de Tucson.

Otro de los aspectos característicos del sistema de planeamiento territorial en Arizona, es que los planes comprehensivos de los condados no supeditan a los planes de ciudades y pueblos como el de la aglomeración urbana de la ciudad de Tucson. Por tanto el plan comprehensivo del condado de Pima, Pima Prospers 2015, no condiciona al Plan Tucson 2013 pero, de acuerdo con las 
respuestas abiertas a los cuestionarios realizados en ambas administraciones, sí que se ha contado estrechamente con el gobierno del condado de Pima, así como a través del proceso de participación pública del Plan Tucson 2013, aunque no tuviera autoridad sobre este último.

Por otro lado, el uso de los indicadores de seguimiento para la evaluación de los planes comprehensivos está bastante extendido. Estos indicadores se recopilan anualmente para obtener la información necesaria para la elaboración de informes anuales y para la renovación del plan a los diez años. Uno de los indicadores más destacado, utilizado como indicador sintético por si mismo, es la medición del consumo de suelo. La unidad de medida de consumo de suelo anual es el indicador clave de seguimiento. Además, está directamente relacionado con las medidas de implementación de estrategias para la conservación del suelo que existen. Por otra parte, la experiencia indica que los datos continuos pueden ser problemáticos de obtener y generalmente requieren tratamientos estadísticos para dar solución a sus carencias. En el caso del Plan Tucson 2013, se incorpora la implementación y la medición de la consecución de objetivos con indicadores como apuesta ambiciosa. Como se corrobora con las entrevistas técnicas realizadas en la ciudad de Tucson, el uso de indicadores implica compromisos y responsabilidad que deben ser asumidos y aceptados políticamente. Por todo ello, se ha hecho un esfuerzo por incorporar el proyecto piloto STAR con más de 600 indicadores que actúan como medidores de gran interés, pero sin compromisos ni responsabilidades legales.

Igualmente interesantes son los procesos de participación ciudadana que se han llevado a cabo tanto en el Plan Tucson 2013 como en Pima Prospers 2015. En ambos instrumentos se ha ido más allá de las exigencias legales mínimas. En ambos casos, sobre todo destacan las numerosas audiciones públicas para los nuevos grandes desarrollos y las propuestas de modificaciones substanciales de los planes anteriores. Por ejemplo, según los entrevistados del departamento de planificación de la ciudad de Tucson, el Plan Tucson es de abajo-arriba (bottom-up), es decir, ha sido desarrollado a lo largo de un prolongado e intenso proceso donde la participación ciudadana ha sido el eje central del mismo (Tabla 3). Se ha trabajado a nivel de barrios, con ONG, con la Cámara de Comercio y otros actores territoriales importantes. En realidad ha existido un verdadero diálogo entre todos los actores territoriales sobre las diferentes políticas a llevar a cabo en el plan.

Por ejemplo, una de las propuestas surgidas desde la participación ciudadana durante las jornadas de trabajo con la comunidad fue incorporar la creación de huertos urbanos para este nuevo Plan Tucson 2013. Además, este plan se presenta con un formato amigable y entendible para todos los ciudadanos que facilita la participación. Desde el Departamento se asegura que se ha tratado de un intercambio del que se ha aprendido mucho por parte de todos aunque se ha reconocido el peso superlativo de los actores económicos. 
Tabla 5. Proceso de participación pública del Plan Tucson 2013

\begin{tabular}{|c|c|c|c|c|c|c|c|}
\hline $\begin{array}{l}\text { Prim } \\
20\end{array}$ & avera & $\begin{array}{c}\text { Verano } \\
2011\end{array}$ & $\begin{array}{l}\text { Otoño } \\
2011\end{array}$ & $\begin{array}{c}\text { Primavera } \\
2012\end{array}$ & $\begin{array}{l}\text { Otoño } \\
2012\end{array}$ & $\begin{array}{c}\text { Ratificación } \\
2013\end{array}$ & Referéndum \\
\hline $\begin{array}{l}\text { Audiciones } \\
\text { públicas } \\
\text { iniciales }\end{array}$ & $\begin{array}{l}\text { Reuniones: } \\
\text { orientación } \\
\text { de actores } \\
\text { territoriales }\end{array}$ & $\begin{array}{l}\text { Grupos de } \\
\text { trabajo con } \\
\text { actores } \\
\text { territoriales }\end{array}$ & $\begin{array}{l}\text { Foros de } \\
\text { participación } \\
\text { y propuestas } \\
\text { con actores } \\
\text { territoriales }\end{array}$ & $\begin{array}{c}\text { Jornadas de } \\
\text { trabajo con } \\
\text { la } \\
\text { comunidad }\end{array}$ & $\begin{array}{l}\text { Sesiones de } \\
\text { revisión y } \\
\text { comentarios } \\
\text { finales }\end{array}$ & $\begin{array}{c}\text { Esfuerzos en } \\
\text { información y } \\
\text { educación } \\
\text { ciudadana }\end{array}$ & $\begin{array}{c}\text { Día de las } \\
\text { elecciones } \\
(5 / 11 / 2013)\end{array}$ \\
\hline \multicolumn{6}{|c|}{ FASE DE ELABORACIÓN DEL BORRADOR DEL PLAN } & $\begin{array}{l}\text { PLAN } \\
\text { FINAL }\end{array}$ & \\
\hline
\end{tabular}

Fuente: elaboración propia a partir de Plan Tucson 2013, Apéndice B

Por último, merece la pena destacar como proceso participativo y democrático el referéndum que implica la aprobación, renovación o modificación substancial de un plan comprehensivo en ciudades y pueblos de Arizona. Esta ratificación democrática para los planes de ordenación de ciudades y pueblos supone una presión añadida para los polííicos, e incluso para los técnicos, que no se exige en el caso de los planes del Condado. Además, la credibilidad social de la planificación, tras un largo proceso participativo, puede verse mermada ante un resultado negativo en el referéndum. Al mismo tiempo, la ratificación por el conjunto de la sociedad podría culminar el proceso de planificación territorial más democrático y consensuado.

\section{Conclusiones y lecciones aprendidas}

La experiencia práctica de la planificación territorial americana se ha enriquecido mucho gracias a la tradición localista anglosajona. En gran parte esto se pone de manifiesto en el desarrollo de modelos colaborativos y comunicativos de planificación. El diálogo basado en proyectos territoriales y la modelización de escenarios utópicos, elaborados gracias a programas informáticos, facilitan la participación ciudadana y el entendimiento de los complejos problemas territoriales. Sin duda este mayor entendimiento e importancia dada a la formación de redes de conocimiento extendidas ayudan en la búsqueda de un proyecto territorial consensuado que de respuesta a los problemas territoriales complejos.

El caso del condado de Pima es una referencia interesante para la ordenación del territorio en Europa y en España. La amplia experiencia planificadora, de hasta tres generaciones de planes, demuestra también la complejidad que engloba la práctica de la planificación territorial y lo mucho que queda por recorrer en España donde todavía muchos planes territoriales no han sido renovados. Por otra parte, se han detectado las inercias institucionales y la defensa de sus competencias propias como unas de las principales cuestiones que frenan la capacidad de coordinación y cooperación para poder llevar a cabo una gestión inteligente e integrada del territorio. La experiencia positiva de cooperación y colaboración interdepartamental del condado de 
Pima y de la ciudad de Tucson podrían servir de ejemplo. Mejorar en materia de coordinación y cooperación administrativa requiere tiempo y experiencia, liderazgo y voluntad política, así como un necesario y verdadero cambio socio-institucional profundo a favor de una visión global e integradora del territorio.

Los problemas de implementación de los planes comprehensivos americanos son solventados en gran parte gracias a su mayor flexibilidad y carácter estratégico. Ello facilita la aceptación de las responsabilidades por cada una de las partes pertinentes. Este modelo se aleja del modelo racional de planificación, excesivamente tecnocrático, buscando principalmente la capacidad de acuerdo de los actores territoriales y fortaleciendo la participación ciudadana durante todo el proceso de planificación. En la nueva planificación territorial de carácter estratégico el técnico planificador debe encontrarse inmerso, en un proceso de aprendizaje continuo que le acerque a un rol principal como mediador. Por último, la consolidación democrática de la planificación territorial a través de las urnas, lejos de ser el enemigo de técnicos y políticos, debe ser defendida como el premio, la garantía y el reconocimiento social de un proyecto de futuro territorial común.

Agradecimientos: Este artículo se sustenta en los trabajos realizados en el marco del proyecto europeo SWAN (Ref.: 7th FP European Grant Agreement N 294947, FP7-INCO-2011-7) y gracias a una estancia de investigación de cuatro meses de duración en la ciudad de Tucson (Arizona, EEUU), financiada por la convocatoria de Movilidad del Programa de Formación del Profesorado Universitario del Ministerio de Educación, Cultura y Deporte (Becas FPU).

Declaración responsable: El autor declara que no existe ningún conflicto de interés en relación a la publicación de este artículo. 


\section{Bibliografía}

Anderson, L. (1995). Guidelines for Preparing Urban Plans. Chicago: American Planning Association Planners Press.

APA (2002). Growing Smart Legislative Guidebook 3: Model Statutes for Planning and the Management of Change. Chicago: APA Planners Press.

Bassett, E. (1938). The master plan, with a discussion of the theory of community land planning legislation. New York: Russell Sage foundation.

Bassett, E. (1940). Zoning. New York: Russell Sage foundation.

Berke, P. R., \& Manta Conroy, M. (2000). Are We Planning for Sustainable Development? An evaluation of 30 comprehensive plans. Journal of the American Planning Association, 66, 21-33.

Campbell, S., Y Fainstein, S. S. (Eds.). (2012). Readings in planning theory (3 rd., ed). Oxford. Wiley-Blackwell.

Cardwell, H., Langsdale, S., \& Stephenson, K. (2009). The Shared Vision Planning Primer: How to incorporate computer aided dispute resolution in water resources planning. Institute for Water Resources (USACE). Retrieved from http://www.sharedvisionplanning.us/

Cullingworth, B. J., \& Caves, R. (2013) [1997]. Planning in the USA: Policies, Issues, and Processes London \& New York: Routledge.

Dandekar, H., \& Main, K. (2014). Small-town comprehensive planning in California. In N. Gallent \& D. Ciaffi (Eds.), Community action and planning (pp. 157-176). Policy Press Scholarship Online.

Davoudi, S., \& Madanipour, A. (2015). Reconsidering Localism. London \& New York: Routledge.

Ellin, N. (2010). Canalscape: Practising Integral Urbanism in Metropolitan Phoenix. Journal of Urban Design, 15, 599-610.

Espluga, J., \& Subirats, J. (2007). Participación ciudadana en las políticas de agua en España. Fundación Nueva del Cultura Agua. Retrieved from http://www.fnca.eu/images/documentos/politica/informes/Participaci\%C3\%B3n\%20ciudad ana\%20en\%20las\%20pol\%C3\%ADticas\%20de\%20agua\%20en\%20Espa\%C3\%B1a.pdf

Fainstein, S. S. (2010). The Just City. Ithaca. New York: Cornell University Press.

Faludi, A. (2010). Cohesion, coherence, co-operation: European spatial planning coming of age? London \& New York: Routledge.

Forester, J. (2013). Planning in the face of conflict: the surprising possibilities of facilitative leadership. Chicago: APA Planners Press. 
Gao, J., Kornov, L., \& Christensen, P. (2013). Do indicators influence communication in SEA? Experience from Chinese practice. Environmental Impact Assessment Review, 43, 121-128.

Gottmann, J. (1961). Megalopolis. The urbanized northeastern seaboard of the United States. New York: The Twentieth Century Fund.

Hallaq, M. (2014). Constitución de los Estados Unidos, carta de derechos, las enmiendas, federalistas y más (Annotated Spanish edition). Government of the United States of America.

Haughton, G., \& Allmendinger, P. (2013). Spatial Planning and the New Localism. Planning Practice \& Research, 28(1), 1-5. Retrived from

https://doi.org/10.1080/02697459.2012.699706

Hernández-Mora, N., \& Ballester, A. (2011). Public participation and the role of social networks in the implementation of the Water Framework Directive in Spain. Ambientalia, Revista Interdisciplinar de Ciencias Ambientales, 20. Retrieved from http://hdl.handle.net/10481/21688

Hoch, C. (2016). Utopia, scenario and plan: A pragmatic integration. Planning Theory, 15, 6-22.

Howard, E. (2010) [1898]. To-morrow: a peaceful path to real reform. Cambridge: Cambridge University Press.

Howard, E. (1985) [1902]. Garden cities of tomorrow. Eastbourne, UK: Orion.

Kelly, E. D. (2010). Community Planning: An Introduction to the Comprehensive Plan. Washington D.C.: Island Press.

Kelly, E. D., \& Becker, B. (2000). Community planning: an introduction to the comprehensive plan. Washington D.C.: Island Press.

Kent, T. J. (1964). The urban general plan. San Francisco: Chandler Pub. Co.

Lauber, T. B., Decker, D. J., \& Knuth, B. A. (2008). Social networks and community-based natural resource management». Environmental management, 42, 677-687.

Leccese, M., \& Mccormick, K. (2000). Charter of the new urbanism. New York: McGraw-Hill.

Mcharg, I. L. (1995) [1969]. Design with Nature. New York: Wiley.

Mohammadreza Talakesh, S., Mohammadi, J., \& Zangiabadi, A. (2015). Comprehensive Planning of Urban Housing in Metropolises by Focusing on Urban Integrated Management. Modern Applied Science, 9(13). https://doi.org/10.5539/mas.v9n13p22

Moss, T. (2012). Spatial Fit, from Panacea to Practice: Implementing the EU Water Framework Directive. Ecology and Society, 17. https://doi.org/10.5751/ES-04821-170302

Mumford, L. (1989) [1961]. The city in history: its origins, its transformations, and its prospects. San Diego \& New York \& London: Harcourt. 
Needham, B. (2006). Planning, Law and Economics: The Rules We Make for Using Land. Abingdon, Oxon \& New York: Routledge.

Needham, B., \& Hartmann, T. (2016). Planning By Law and Property Rights Reconsidered. Abingdon, Oxon \& New York: Routledge.

O'toole, K., Wallis, A., \& Mitchell, B. (2009). Place-based knowledge networks: the case of water management in South-West Victoria, Australia. Water Alternatives, 2, 101-114.

Pahl-Wostl, C. (2007). The implications of complexity for integrated resources management». Environmental Modelling \& Software, 22, 561-569.

Paris, C. (2013). Critical Readings in Planning Theory: Urban and Regional Planning Series. Pergamon: Elsevier.

Pendall, R., \& Puentes, R. (2008). Land-use regulations as territorial governance in U.S. metropolitan areas. Boletín de la Asociación de Geógrafos Españoles, 46, 181-206. Retrieved from https://www.age-geografia.es/ojs/index.php/bage/article/view/682/636

Peterson, G. D., Cumming, G. S., \& Carpenter, S. R. (2003). Scenario Planning: a Tool for Conservation in an Uncertain World. Conservation Biology, 17, 358-366.

Petrov, L. O., Shahumyan, H., Williams, B., \& Convery, S. (2011). Scenarios and Indicators Supporting Urban Regional Planning. Procedia - Social and Behavioral Sciences, 21, 243-252.

Seasons, M. (2003). Monitoring and Evaluation in Municipal Planning: Considering the Realities. Journal of the American Planning Association, 69, 430-440.

Smith, C. S. (2006). The Plan of Chicago: Daniel Burnham and the remaking of the American city. Chicago: University of Chicago Press.

Soja, E. W. (2008). Postmetrópolis: estudios críticos sobre las ciudades y las regiones. Madrid: Traficantes de Sueños.

Soja, E. W. (2011). Postmodern Geographies: The Reassertion of Space in Critical Social Theory $\left(2^{\text {nd }}\right.$ ed.). London: Verso.

Stead, D. (2013). Convergence, Divergence, or Constancy of Spatial Planning? Connecting Theoretical Concepts with Empirical Evidence from Europe. Journal of Planning Literature, 28(1), 19-31. https://doi.org/10.1177/0885412212471562

Talen, E. (2005). New Urbanism and American Planning: The Conflict of Cultures. Abingdon, Oxon \& New York: Routledge.

Widener, M. N. (2013). Moderating Citizen 'Visioning' in Town Comprehensive Planning: Deliberative Dialog Processes. Wayne Law Review, 59, 25-47. 
Zinn, J. A. (2004). State Policies to Manage Growth and Protect Open Spaces. New York: Nova Publishers.

\section{Lista de planes y documentos normativos}

A Standard State Zonning Enabling Act 1926 (Bill by the U.S. Chamber of Commerce). Retrieved from https://www. planning.org/growingsmart/enablingacts.htm

A Standard City Planning Enabling Act 1928. Government of the United States of America. Retrieved from https://www.planning.org/growingsmart/enablingacts.htm

Coastal Zone Management Act Of 1972. Gobierno de Estados Unidos (Public Law 92-583, 86 Stat. 1280 §).

Clean Water Act 1977. Government of the United States of America (Sec. 208 [33 U.S.C. 1288]). Federal Water Pollution Control Act 1948 (Clean Water Act of 1948). Gobierno de Estados Unidos. (33 U.S.C. 1251 - 1376; Chapter 758), P.L. 845, June 30, 1948; 62 Stat. 1155).

Growing Smarter Act 1988 (State of Arizona). Retrieved from http://www.azleg.state.az.us/legtext/45leg/2r/bills/hb2601p.htm

Growing Smarter Plus Act 2000 (State of Arizona). Retrieved from http://www.azleg.state.az.us/legtext/45leg/2r/bills/hb2601p.htm

National Environment Policy Act 1969 (Public Law 91-190, 83 Stat. 852 §).

National Historic Preservation Act 1966. Government of the United States of America (Public Law 89-665, 80 Stat. 915 §).

Oregon's Statewide Planning Goals \& Guidelines (1973) (2010 ed.). Salem, Oregon: Department of Land Conservation and Development. Retrieved from http://www.oregon.gov/LCD/docs/goals/compilation_of_statewide_planning_goals.pdf

Pima Prospers 2015. Comprehensive plan initiative. Pima County. Arizona. Retrieved from http://webcms.pima.gov/government/pima_prospers/

Plan Tucson 2013. City of Tucson. Retrieved from https://www.tucsonaz.gov/pdsd/plan-tucson

Strategic Plan Of Oregon 2014-2022 (2014). Department of Land Conservation and Development. Retrieved from: http://www.oregon.gov/LCD/docs/publications/StrategicPlan2014-22_Draft.pdf The Housing Act 1954. Government of the United States of America (Public Law 83-560, 68 Stat. 590 §). 